\title{
LA DIALÉCTICA DEL DESEO EN LA FENOMENOLOGÍA DEL ESPÍRITU DE HEGEL
}

Carlos Emel Rendón*

Resumen: En el presente artículo intentamos una reconstrucción del significado que presenta para Hegel el fenómeno del deseo en el contexto de la exposición de la figura de la "Autoconciencia" en la Fenomenología del Espiritu. Defendemos la tesis de que el deseo representa propiamente la esencia negativa de la autoconciencia, ya que es en tanto deseante como ella pretende alcanzar la afirmación de sí frente a un mundo -natural y humano- que carece de independencia y sentido propios. Con este fin, nos ocupamos de determinar la naturaleza dialéctica que caracteriza al sujeto deseante frente a la otredad y diferenciamos tres momentos fundamentales en la estructura del deseo: desde las experiencias más inmediatas o naturales hasta las más elaboradas o espiritualizadas, las que se resuelven en el deseo de reconocimiento como deseo ya no de un "objeto" sino de otro "yo".

Palabras clave: Hegel, deseo, autoconciencia, negación, objeto, satisfacción

Abstract: In this paper we attempt a reconstruction of the meaning that presents for Hegel the phenomenon of desire in the context of the exposition of the figure of "self-consciousness" in the Phenomenology of Spirit. We defend the thesis that the desire itself represents the negative essence of self-consciousness, and it is as desirous as it aims to achieve self-assertion in a -natural and human- world lacking of independence and own sense. To this end, we determine the dialectic nature that characterizes the desiring subject against otherness and we differentiate three key moments in the structure of desire, from the most immediate or natural experiences to the most elaborate or spiritualized, which are resolved in the desire for recognition as the desire not of an "object" but another "self".

Key words: Hegel, desire, self-consciousness, negation, object, satisfaction

* Doctor en Filosofía por la Universidad de Antioquia (Medellín), Colombia. Profesor Asociado de la Universidad Nacional de Colombia - Sede Medellín. Dirección electrónica: crendona@unal.edu.co 
Uno de los conceptos que más atraen y concentran la atención de cualquier lector de la sección B de la Fenomenología del Espíritu, ${ }^{1}$ que trata sobre la "Autoconciencia", es el concepto del "deseo"2 ("Begierde"). Y es que, además de la fuerza plástica de su exposición, llama la atención la significación que Hegel le confiere para efectos de la formación del concepto de la autoconciencia. En vista de esto último podría decirse que la concepción hegeliana de la autoconciencia lejos de ser pensada en la forma idealista-trascendental de un yo que se abstrae de toda condición espaciotemporal y que se fija autárquicamente en el ámbito que describe la representación pura de sí o la intuición absoluta-, se erige sobre la perspectiva - fundamentalmente práctica- de un Sí mismo necesitado de afirmación identificación y autonomía en las diferentes relaciones que establece con los objetos y los seres del mundo, con lo otro de sí mismo. Este proceso de afirmación, que implica desde las formas más instintivas o naturales, hasta las más reflexionadas o conscientes, es, precisamente, lo que Hegel llama “deseo". Tal es la razón por la cual caracteriza de entrada la autoconciencia como "deseo en general". 3

La autoconciencia es "deseo en general", porque el deseo representa el comportamiento o actitud instintiva-natural, pero también reflexionada, a través de la cual el yo busca afirmarse como la "verdad" o "esencia" en toda relación que establece con la otredad, a la que, sea cual sea la forma en que se presente, entiende como lo negativo de sí. En este comportamiento se

1 Para este trabajo hemos empleado la versión alemana: G. W. F. Hegel. Phänomenologie des Geistes. Edición de Hans-Friedrich Wessels y Heinrich Clairmont. Hamburg, Meiner, 2006. La traducción de los pasajes citados se hará no obstante, cuando no se indique lo contrario, de la versión castellana de W. Roces: G. W. F. Hegel, Fenomenología del Espiritu, México, Fondo de Cultura Económica, 2002. Las citas indicarán el número de páginas de ambas versiones.

2 Hemos optado por traducir la expresión alemana "Begierde" por "deseo", en lugar de "apetencia" (como lo hace la traducción de Roces), teniendo en cuenta que, frente a la apetencia, el deseo encierra, como lo muestra su desarrollo en la Fenomenología del Espiritu, momentos que van más allá del carácter inmediato, preconsciente, que encierra el simple apetito. (Seguimos así también la traducción que hace Valls Plana de "Begierde" en su versión castellana de Enciclopedia de las Ciencias Filosófica. Cf. G. W. F. Hegel, Enciclopedia de las Ciencias Filosóficas, Madrid, Alianza, 1997, p. 476).

${ }^{3}$ Hegel, Phänomenologie des Geistes, p. 121; v. cast.: p. 108. 
encierran al menos, tres sentidos fundamentales del deseo, relacionados entre sí, a saber: el deseo como deseo de unidad consigo, que se expresa en el instinto de la autoconservación; el deseo como deseo de un otro diferenciado en la forma de un objeto del mundo sensible que se expresa en el impulso consciente a la afirmación de la propia identidad; y, finalmente, el deseo como deseo de un otro en la figura propia de un yo, o deseo de reconocimiento. Ahora bien, estos diferentes sentidos, lejos de presentarse aislados e inconexos en el proceso de autoafirmación del sí mismo, remiten uno al otro en una progresiva transición dialéctica, en cuyo curso el sujeto accede a una comprensión más acabada de sí mismo como sujeto deseante y de la naturaleza del objeto deseado. Ello permite entender el deseo y, con éste, el saber de sí que comporta, como implicados en una única unidad experiencial sobre la que descansa, para Hegel, el "concepto" de la autoconciencia. ${ }^{4}$

En la interpretación del deseo que a continuación vamos a esbozar, queremos afirmar, a modo de tesis general, que, para la comprensión de la concepción hegeliana del deseo se requiere de una discriminación de los sentidos o fases que el desear entraña, en la medida en que tales sentidos o fases obedecen a objetos distintos e irreductibles del deseo y a que en ellas se concreta una determinada experiencia del deseo. Para ello intentaremos, en primer lugar, determinar el sentido de la caracterización de la autoconciencia como "deseo en general" (I); con base en ello buscaremos, en segundo lugar, determinar los diferentes sentidos estructurales del deseo arriba señalados, así como el tipo de experiencia del deseo que el sujeto deseante consuma en cada uno de ellos (II).

${ }^{4}$ En este sentido el deseo es en Hegel, como ha observado Förster, "un concepto epistemológico, no psicológico". Cf. Förster, Eckart, "Hegels 'Entdeckungsreisen', Entstehung und Aufbau der Phänomenologie des Geistes", en: Vieweg, Klaus/Welsch, Wolfgang (eds), Hegels Pbänomenologie des Geistes. Ein kooperativer Kommentar zu einem Schlïsselwerk der Moderne, Frankfurt, Suhrkamp, 2008, p. 42, nota 9. 
I.

Lo primero que hemos de intentar aclarar es el sentido de la caracterización de la "Autoconciencia" como "deseo en general". 5 Esta caracterización cae en un contexto en el que Hegel, sintetiza el movimiento conceptual que conduce a $-\mathrm{y}$ del cual es resultado- la nueva "figura del saber", es decir, el "saber de sí" o "Autoconciencia". Hegel, en efecto, habla allí de la autoconciencia sobre el trasfondo de la oposición que tenía lugar bajo la figura de la conciencia sensible, la oposición conciencia-objeto, y se dispone a mostrar el significado que dicha oposición tiene ahora para la conciencia en tanto que autoconciencia. Mientras que, como conciencia, el objeto (el mundo sensible en general) se mantenía frente a ella en una especie de subsistencia autónoma y aparecía como la verdad de su saber, ahora, bajo la figura de la autoconciencia, el objeto se debilita en esta significación, en la medida en que su subsistencia (su verdad) no reside en sí mismo sino en relación con la autoconciencia, la cual pasa a ser ahora, por tanto, la verdad de esta relación. Este cambio en la relación de la conciencia con el objeto se había prefigurado, como se sabe, con la experiencia que la conciencia, bajo la figura del "Entendimiento", había hecho con el objeto en tanto que "fenómeno": la conciencia, como entendimiento, pudo penetrar en el "interior" del fenómeno y encontrarse a sí misma en este interior. ${ }^{6}$ Ella pudo descubrir entonces que su saber de lo otro -el "fenómeno"- era, en el fondo, saber de sí misma y que ella, en tanto es la unidad constitutiva de dicha interioridad, es la verdad del objeto. La oposición entre ella y el objeto, o entre su saber y su verdad, no desaparece, ciertamente, pero el sentido de la misma es ahora esencialmente otro: "Esta contraposición entre su fenómeno y su verdad sólo tiene por esencia la verdad, o sea, la unidad de la conciencia consigo mismo; esta unidad tiene que llegar a ser esencial a

${ }^{5}$ Sobre este tópico véase el exhaustivo análisis que lleva a cabo R. Pippin en su estudio: Hegel on selfconsciousness. Desire and Death in the Phenomenology of Spirit, Princeton University Press, 2011, especialmente capítulo 1, pp. 6-53.

${ }^{6}$ Cf. Bowman. "Kraft und Verstand. Hegels Übergang zum Selbstbewusstsein in der Phänomenologie des Geistes", en: Vieweg, Klaus/ Welsch, Wolfgang (eds), Hegels Phänomenologie des Geistes. Ein kooperativer Kommentar zu einem Scblüsselverk der Moderne. Frankfurt, Suhrkamp, 2008. 
la autoconciencia...". ${ }^{7}$ La unidad de la autoconciencia consigo misma es, pues, "ahora", "la verdad" de aquella oposición.

Es en este contexto donde nos encontramos, de manera un tanto intempestiva, con la definición de la autoconciencia como "deseo en general": tras afirmar que la "unidad [consigo misma] tiene que llegar a ser esencial a la autoconciencia", Hegel añade a continuación: "es decir, ella es deseo en general" ["Begierde überbaupt"]. ${ }^{8}$ ¿Quiere decir ello que el deseo es la conciencia misma (o el sentimiento) de la unidad como lo propiamente esencial al sujeto deseante? Tal es, en efecto, el sentido que parece desprenderse de la frase con que Hegel concluye el pasaje: Hegel da a entender que lo que define a la autoconciencia como autoconciencia es la conciencia de la unidad consigo como lo esencial de ella y -así se desprende del giro "es decir"- llama a esta conciencia "deseo", más concretamente, "deseo en general". En esta caracterización no se trata, sin embargo, meramente de una descripción del deseo como un predicado o cualidad de la autoconciencia: en ella se apunta al hecho de que la autoconciencia es, en sí misma, deseo, pues es bajo esta forma como se comporta en toda relación u "oposición" que establece con lo otro de ella. Dicho de otra manera: dado que en toda relación u oposición lo esencial para ella "tiene que ser" la unidad consigo ella es, "en general", deseo.

Sobre el trasfondo de este contexto puede caracterizarse entonces el comportamiento que la autoconciencia asumirá ante el objeto deseado. Tal comportamiento implica de entrada dos variaciones fundamentales: por un lado, el cambio de significado del objeto para la autoconciencia: el objeto no es ya para ella, como lo era en cuanto conciencia, la "verdad" de su "saber", sino algo que ella, en palabras de Hegel, "marca" con "el carácter de lo negativo": 9 en cuanto tal, o, lo que es lo mismo, en cuanto objeto del deseo, es algo destinado a la "aniquilación"; por otro lado, el sujeto no se comporta ya frente al objeto como frente a algo dado, ya no es el sujeto meramente receptor de la conciencia sensible, sino, en cuanto sujeto deseante, es un sujeto actuante, práctico, en el sentido de que él mismo, como lo asevera

${ }^{7}$ Hegel, Phänomenologie des Geistes, op. cit., p. 121; v. cast. p. 108.

${ }^{8}$ Ibid., p. 121; Roces traduce: "ésta [la autoconciencia] es, en general, apetencia”: cf. v. cast. p. 108.

${ }^{9}$ Cf. Ibid., p 121-122; v. cast. p. 108. 
Hegel, es el "movimiento" "en" el cual tiene lugar la superación de la oposición entre él mismo y el objeto. ${ }^{10}$ Mientras que el primer aspecto representa un cambio de paradigma en la relación sujeto-objeto, motivado por la significación negativa que el objeto tiene para la autoconciencia, el segundo representa un cambio en el orden experiencial de la conciencia, en la medida en que el deseo - que ella es- le lleva a desplegar actividades que le permitan afirmarse como lo esencial frente al objeto del deseo. Se trata de dos aspectos que se implican mutuamente: el objeto del saber deviene objeto del deseo porque, con la aparición del nuevo comportamiento de la conciencia, el objeto ha entrado en la significación práctica de ser medio de gozo y satisfacción de dicha conciencia; ésta, a su vez, deviene autoconciencia deseante porque sólo como "deseo" puede llevar a cabo la experiencia de la significación con la que "marca" el objeto. Ambos aspectos, así como la implicación recíproca que guardan entre ellos, se pondrán de manifiesto con los tres sentidos del deseo antes aludidos.

\section{II.}

Una vez delimitado el significado de la caracterización de la autoconciencia como "deseo en general", es preciso entrar ahora a determinar las formas particulares en que la autoconciencia se comporta como deseante o, lo que es lo mismo, las diferentes experiencias del deseo en que ella intenta afirmarse como ser para sí.

$\mathrm{Si}$, como lo afirma Hegel, la unidad consigo es lo esencial a la autoconciencia y ésta, por ello mismo, es deseo, entonces el objeto inmediato del deseo del sujeto autoconsciente no puede ser otro que él mismo, su propio yo. Decir que la autoconciencia es inmediatamente o en su aparición, deseo de sí, es afirmar que el yo se autoconstituye en el "objeto" exclusivo de todos los esfuerzos o tendencias que le permiten afirmarse como tal yo. Esto significa que la afirmación de sí no se da en forma de una simple representación de sí mismo como tal yo, o del reconocimiento de su identidad en sus diversos estados mentales, sino en la forma de una actividad práctica cuyo telos es el aseguramiento de las condiciones bajo las cuales es posible su permanencia espacio-temporal

${ }^{10}$ Cf. Ibid., p 122; v. cast. p. 108. 
como un "viviente". En esta forma primaria del deseo, se nos pone al descubierto la función que cumple en relación con la conservación de sí: la de que todo proceso de formación de lo que es un "yo" parte necesariamente del aseguramiento, por parte de éste, de las condiciones de su subsistencia o superviviencia. Ésta es, a nuestro juicio, una de las intuiciones fundamentales que subyace a la idea de la vida que Hegel expone en el contexto de la autoconciencia: la de que ya en las fases más primarias del deseo se expresa una clara autocomprensión del sujeto humano como sí mismo independiente.

En esta perspectiva naturalista, la autoconciencia aparece, como el resto de los organismos vivos, tratando de afirmar su "independencia" frente a aquel proceso o "fluidez universal", como lo evidencia esa tendencia, característica de todo lo viviente, a hacer de la vida el medio de satisfacción de las necesidades que ella misma impone. En este sentido habla Hegel de un "negar", por parte de la figura viviente que es "para sí", la "fluidez y continuidad de la vida", de un "afirmarse" como no disuelto en este universal", y de un "mantenerse" "al disociarse de esta su naturaleza inorgánica" y al "devorarla".11 Mas lo propio de este deseo inmediato no es la sola "destrucción" o consumo del objeto con miras a la conservación de sí como organismo viviente; hay también en ello -y eso es lo que expresa el conatus de separación de su "naturaleza inorgánica"- un intento por conservarse en la forma de aquella "figura independiente" bajo la cual el viviente-yo se concibe -y opone- frente a la vida como faktum de la naturaleza. En efecto, mientras que la vida es independiente "en sí", esto es, como objeto o "sustancia", la autoconciencia es independiente porque es, simplemente, "para sî":12 su independencia (Selbständigkeit) es, por ende, el objeto mismo de su conatus de conservación, y es con vistas a ella como habría que entender la búsqueda de la satisfacción de las necesidades y funciones que emanan de su propia naturaleza biológico-animal, o, dicho con Brandom, de su naturaleza "erótica"."13

${ }^{11}$ Ibid., pp. 123-124; v. cast. p. 110.

12 Cf. Ibid., p. 122; v. cast. p. 109.

${ }^{13}$ Cf. Brandom, Robert B. "Selbstbewusstsein und Selbst-Konstitution", en: Halbig, Christoph /Quante, Michael/ Siep, Ludwig (eds), Hegels Erbe, Frankfurt, Suhrkamp, 2004 , p. 54. Esta estructura "erótica" se expresaría en las "relaciones" de "hambre", "comida" y "alimentación". 
Es evidente que el deseo de autoconservación no se agota en la simple apropiación o consumo de lo viviente, sino que se resuelve en una estrategia de diferenciación y afirmación de la propia independencia frente al objeto mismo del deseo, encarnado en la vida como "proceso". Dado que, bajo esta forma, el deseo de afirmación está condicionado por el "proceso" de la vida $y$, en este sentido, la pretensión de afirmación no puede elevarse por encima de las condiciones que determinan el lugar y la situación del viviente frente a dicho proceso, la tendencia consciente a la afirmación debe ir más allá del círculo que describe la propia naturalidad y abrirse paso hacia formas del deseo no escamoteadas por la inagotabilidad e infinitud propias de la vida.

Por ello mismo, el siguiente paso en la dialéctica del deseo lo representa justamente la apropiación de la conciencia de su "yo puro" mediante la negación de lo otro de sí: la experiencia de la vida como objeto independiente e inagotable se trueca ahora en la experiencia de un otro singularizado, en el que la autoconciencia pueda consumar la negatividad que ella es en tanto yo "simple" e "inmediato ser para sí". Podemos entender este paso como el paso hacia la individuación del objeto del deseo, en la medida en que el objeto deseado no se presenta ahora para el yo como un todo inconmensurable, sino como un objeto singular, constitutivo, como el propio sujeto deseante, de aquel todo. Dicha individuación del objeto del deseo conlleva por ello un cambio esencial en el comportamiento de la autoconciencia deseante: ésta tiende a afirmar ahora la certeza originaria de sí misma de ser puro ser para sí. En este movimiento de afirmación el yo se comporta como "esencia negativa"14 en el sentido de ser la negación de lo otro de sí.

Hegel describe en estas palabras lo que podría considerarse como el pathos de la esencia negativa que es la autoconciencia deseante: "Cierta de la nulidad de este otro pone para sí esta nulidad como su verdad, aniquila el objeto independiente y se da con ello la certeza de sí misma como verdadera certeza."15 El pathos mismo de la autoconciencia deseante no es otra cosa que esta tendencia consciente a la negación de lo deseado, porque, a los ojos del sujeto que desea, el objeto carece en sí de significación: lo que importa

${ }^{14}$ Cf. Pbänomenologie des Geistes, p. 122; v. cast. p. 109.

${ }^{15}$ Idem. 
ante todo es la satisfacción, el goce. Lo que Hegel llama la "nulidad del serotro" es precisamente la carencia de valor que tiene para el sujeto deseante el objeto del deseo cuando se lo considera más allá de la esfera del deseo. Lejos de ver el objeto del deseo como un "en sí", portador de un valor irreductible e inalienable, el sujeto deseante ve el objeto como algo que sólo es en la medida en que es deseado. Nada son en sí mismos el alimento, la bebida, el vestido, el dinero, si no hay un sujeto hambriento, un sujeto sediento, un sujeto necesitado de abrigo, o un sujeto que trabaje o intercambie bienes o productos. El deseo confiere al objeto una valoración esencialmente subjetiva, egoísta, si se quiere, por mor de la cual éste aparece como destinado meramente a la afirmación de su poder sobre él, a su satisfacción. Por ello mismo, ésta no puede tener el sentido de un disfrute libre, desinteresado o contemplativo del objeto deseado. $\mathrm{Ha}$ de tenerse presente que la relación del deseo con el objeto es descrita por Hegel en términos de "aniquilación", ${ }_{16}$ y que es la experiencia de la misma lo que constituye, en rigor, la experiencia paradigmática del deseo, en la medida en que es esta experiencia la que torna verdadera la certeza inherente al sujeto deseante, la certeza de que lo propiamente esencial, es el yo que desea, y no el objeto deseado. ${ }^{17}$

16 Ya en el Sistema de la Eticidad (1802-1803), al exponer la naturaleza del "sentimiento práctico" o "goce" en el contexto de la "eticidad natural", Hegel hacía resaltar el carácter negativo del mismo: el goce (Genuss), decía allí, "tiende a la absoluta singularidad del individuo y, por ende, a la destrucción de lo objetivo y lo universal"; en él, el objeto "es completamente aniquilado" y, por lo mismo, es goce "puramente sensible"(Hegel, System der Sittlichkeit, Hamburg, Meiner, 2002. p. 7). Lo que llama la atención en esta reflexión precedente es el hecho de que ya allí el goce es visto en la perspectiva de un comportamiento que, si bien provee al sujeto satisfecho la "unidad" o el "sentimiento de sí", no obstante se muestra en sí mismo como necesitado de superación, toda vez que de goce "sensible" ha de convertirse en goce "ético" o "racional", lo cual supone un cambio en el comportamiento del sujeto frente al objeto (comportamiento que en el Sistema de la Eticidad se expresa en el "trabajo", el "producto y la posesión" y la "herramienta"). Esta necesidad de superación del goce sensible se encuentra nuevamente en la Fenomenología del Espiritu en la transición dialéctica del deseo del objeto al deseo de otra autoconciencia.

17 Esta connotación del deseo, que resulta comprensible si se lo entiende como expresión de la "esencia negativa" que es la autoconciencia deseante, encontrará su expresión radical en la figura del "señor": frente a su deseo, no sólo la cosa, sino 
Ahora bien, esta suerte de prelación o dominio del yo deseante sobre el objeto se explicaría por esa especie de supremacía ontológico-epistémica que el yo se atribuye a sí mismo en su relación u oposición con el objeto, en la medida en que, por un lado, la "verdad" de esta oposición es, como se vio antes, la "unidad de la autoconciencia consigo misma", y por otro, esta verdad es la "esencia" de la autoconciencia; tal supremacía, por lo demás, se corresponde enteramente con aquella nulidad ontológica que encarna el objeto para el sujeto deseante. Lo que constituye, por tanto, el propósito esencial, consciente de sí, del sujeto deseante en la satisfacción del deseo es la conservación de esta supremacía como condición de su autoaseguramiento como sí mismo "independiente". ¿Quiere decir ello que la satisfacción del deseo representa el momento culminante de la experiencia de la "independencia" de la autoconciencia, de esa independencia que, en efecto, es constitutiva de su concepto y bajo la cual ella aparece y se relaciona con el ser-otro?

Esta cuestión nos conduce a la paradoja esencia que advierte Hegel en la naturaleza del humano en general. Expresada en sus palabras, esta paradoja se presenta de la siguiente forma: "El deseo y la certeza de sí misma alcanzada en su satisfacción se hallan condicionadas por el objeto, ya que la satisfacción se ha obtenido mediante la superación de este otro; para que esta superación sea, tiene que ser este otro. Por tanto, la autoconciencia no puede superar al objeto mediante su actitud negativa ante él; lejos de ello, lo reproduce, así como reproduce el deseo."18 Se trata, en el fondo, de una paradoja de doble sentido: por un lado, la autoconciencia deseante se halla condicionada por la presencia real del objeto: éste sólo puede ser superado, si está dado previamente al sujeto que lo requiere; por otro, la satisfacción

también el siervo, que la elabora, carecen en sí de valor ontológico propio: la cosa y el siervo no son nada en sí mismos, sólo son como medios o instrumentos de satisfacción del deseo del señor. Justamente por compartir, como la cosa, el carácter de medio o instrumento, Hegel llegará a caracterizar al siervo como un ser "cosificado". Cf. Fenomenología del Espiritu, p.117. Véase para ello: Kojève, Alexandre, La dialéctica del amo y del esclavo en Hegel, Buenos Aires, Leviatán, 2006, p. 27; cf. Kelly, George Armstrong. "Bemerkungen zu Hegels 'Herrschaft und Knechtschaft", en: H. F. Fulda/D. Henrich (Eds), Materialien zu Hegels 'Phänomenologie des Geistes', Frankfurt, Suhrkamp, 1973, pp. 189ss.

${ }^{18}$ Hegel, Phänomenologie des Geistes, p. 126; v. cast. p. 112. 
del deseo conlleva la reproducción futura del mismo y del objeto, y, con ello, la recaída de la autoconciencia en la dependencia respecto del objeto. La autoconciencia, por tanto, no sólo no parece consolidar su independencia frente al objeto deseado, sino que, por el contrario, su experiencia es lo contrario a lo esperado por ella: la experiencia de la satisfacción del deseo es la experiencia de la independencia del objeto, es decir, la experiencia de que el objeto del deseo es en sí mismo inagotable y de que su negación, o lo que es lo mismo, la satisfacción, ha sido pasajera. Ya el hecho de que el deseo se reproduzca a sí mismo y, reproduzca, con ello, la necesidad del objeto, da cuenta del carácter condicionado y, por tanto, dependiente que marca la experiencia general del deseo. Bajo esta perspectiva, la satisfacción del deseo se revelaría entonces, como señala Honneth, como "ilusoria" y la experiencia del deseo como "insuficiente" o incluso "fracasada". ${ }^{19}$

A esta experiencia paradójica del deseo se suma otra no menos determinante y característica del deseo humano: la experiencia de que lo deseado es un otro y, concretamente, un otro de sí mismo, pues Hegel afirma: "Es, en realidad, un otro que la autoconciencia, la esencia del deseo; y por medio de esta experiencia ha devenido para ella misma esta verdad". ${ }^{20}$ La experiencia de que un "otro que la autoconciencia" es la esencia del deseo es, claramente, una experiencia que desmiente la pretensión fundamental de la autoconciencia, el ser la verdad o la esencia de lo deseado. El objeto ha demostrado ser esta esencia, en la medida en que es la condición de la satisfacción del deseo; con ello, la autoconciencia deseante deviene una autoconciencia extrañada y dependiente: extrañada, porque tiene su esencia o su verdad en un otro esencialmente diferente a ella, encarnado en la figura de un no-yo; dependiente, porque la existencia misma de esto otro representa la condición de posibilidad de su propia afirmación. Bajo esta doble característica de su estructura, el deseo se presenta como una especie de inquietud vital del Sí mismo que nunca alcanza reposo en la satisfacción lograda.

19 Cf. Honneth, "Von der Begierde zur Anerkennung. Hegels Begründung von Selbstbewusstsein", en: Vieweg, Klaus/ Welsch, Wolfgang (eds), Hegels Pbänomenologie des Geistes. Ein kooperativer Kommentar zu einem Scblüsselwerk der Moderne, Frankfurt, Suhrkamp, 2008, pp. 196-198.

${ }^{20}$ Hegel, Pbänomenologie des Geistes, p. 126; v. cast. p. 112. 
Considerada sobre el trasfondo de la dialéctica precedente, esta conclusión viene a significar, positivamente, lo siguiente: la autoconciencia sólo logra su verdadera satisfacción, en relación con un objeto que, a diferencia del objeto meramente sensible, posibilite definitivamente su afirmación como "absolutamente para sí", como independiente. ¿Cómo es posible dicha satisfacción atendiendo a lo que es la naturaleza propia del objeto del deseo, esto es, el ser independiente "en sî" y "negativo"? La respuesta a esta pregunta nos conduce por sí a la tercera fase de la dialéctica del deseo, el deseo de un otro en la figura propia de un yo o deseo de reconocimiento.

Esta nueva fase del deseo se presenta, de hecho, como movimiento de inversión en el proceso de su afirmación como "verdad": dado que el deseo no logra la negación real del objeto y, en consecuencia, no logra la afirmación absoluta del sujeto deseante frente a él, es necesario que la negación se presente como un movimiento propio de lo deseado: la autonegación del objeto - tal es la premisa que motiva el tránsito hacia la nueva fase del deseo- es la condición de la satisfacción de la autoconciencia. Dicho tránsito es explicitado por Hegel de esta manera: "Por razón de la independencia del objeto, la autoconciencia sólo puede (...) lograr satisfacción en cuanto que este objeto mismo cumple en él la negación; y tiene que cumplir en sí esta negación de sí mismo, pues el objeto es en sí lo negativo y tiene que ser para otro lo que él es." 21 Ahora bien, es evidente que una tal negación no puede ser la actividad de un objeto natural cualquiera: en la medida en que se trata de una "negación de sî", se trata de un objeto dotado de reflexión, es decir, de un yo, una "conciencia". Por consiguiente, el que la satisfacción de la autoconciencia deseante se halle sujeta a la condición expresa de que el objeto deseado, es decir, el otro yo, cumpla en sí mismo el movimiento de negación propio del deseo, significa que toda relación entre sujetos autoconscientes es posible sólo bajo la condición de que cada uno sirva al otro como medio de la autorrealización anhelada. ${ }^{22}$ La negación de sí haría alusión no sólo, como lo afirma Honneth

\section{${ }^{21}$ Cf. Idem.}

${ }^{22}$ En este sentido anota Pinkard: "The objects of the world that are not selfconsciuos have no projects; they cannot certify for the subject that his own projects are indeed authoritative. Only another agent with his own projects can do it": 
a un "acto de autolimitación por medio del cual se le revela al sujeto su "dependencia 'ontológica", 23 sino que alude también, y quizá de manera más radical, a una superación del tipo de relación prerreflexiva que encarna el deseo, en virtud de las limitaciones que le son inherentes para efectos del establecimiento de relaciones intersubjetivas logradas o, más concretamente, de reconocimiento. Sólo tras el abandono de la estrategia propia del deseo, a saber, la afirmación de sí mismo a costa de la exclusión o aniquilación de lo otro-deseado, puede darse el paso hacia relaciones o actitudes entre los sujetos erigidas sobre supuestos esencialmente diferentes a los que mueven a las relaciones de índole pragmático-instrumental. Tal es la condición sobre la que Hegel erige el postulado con el que da paso a la nueva figura del deseo, el postulado según el cual, "la autoconciencia sólo alcanza su satisfacción en otra autoconciencia." 24

Este postulado, que marca el tránsito hacia el movimiento del reconocimiento, apuntaría precisamente a lo que es la condición, ya aludida, de esta nueva fase del deseo: la de que la satisfacción de la autoconciencia sólo es posible en la medida en que la otra autoconciencia se niegue en sí misma como aquello que, de otra manera, es un obstáculo a la satisfacción del deseo "en general" (no sólo del deseo de otro yo), es decir, que se niegue como singularidad excluyente o que sólo sabe de sí, en otras palabras, que supere su propio comportamiento como autoconciencia deseante. Para ilustrar el papel que juega este comportamiento en la dialéctica misma del reconocimiento, ${ }^{25}$ bastaría con recrear ligeramente la descripción hegeliana de la relación (unilateral) de reconocimiento del "señor" y el "siervo", pues allí puede verse en qué medida tal relación se debe no sólo al triunfo de una de las autoconciencias sobre la otra en el proceso de la "lucha", sino también a la persistencia de la una en su condición de ser para sí excluyente e inmediato. Tal relación pone de manifiesto, en efecto, que, en tanto relación de dominio, obedece al carácter unilateral que presenta allí el

Pinkard, Terry, Hegel's Phenomenology.The Sociality of Reason, Cambridge University Press, 1996, p. 53.

${ }^{23}$ Cf. Honneth, "Von der Begierde zur Anerkennung. Hegels Begründung von Selbstbewusstsein", loc. cit., pp. 200-202.

${ }^{24}$ Hegel, Pbänomenologie des Geistes, p. 126; v. cast. p. 112.

25 Véase para ello Ludwig Siep, Praktische Pbilosophie im Deutschen Idealismus, Frankfurt, Suhrkamp, 1992, pp. 172 y ss. 
movimiento de la autonegación: es claro que ésta es consumada solamente por la conciencia servil y que su restitución a sí como autoconciencia precisa del cumplimiento de esta negación por parte de la conciencia dominante. En tal sentido, cabe decir que "señor" y "siervo" simbolizan formas contrapuestas de la relación del yo con la propia naturaleza deseante, con la propia negatividad. Tal es lo que se evidencia, en efecto, al final de la exposición de la experiencia de la figura de la conciencia dominante: para que el "reconocimiento en sentido estricto", tenga lugar, es necesario que "lo que el señor haga contra el otro lo haga también contra sí mismo" y que "lo que el siervo hace contra sí lo haga también contra el otro." 26 En el caso particular de la conciencia servil ${ }^{27}$ el cumplimiento de esta condición supondría su reivindicación como autoconciencia sobre la base de las experiencias que la forman en su condición de conciencia dominada (el temor, el servicio y la obediencia), experiencias que, a la vez que consuman el proceso de la negación de sí como autoconciencia inmediata o natural, la disponen a una relación con el ser-otro (el objeto sensible o del deseo) que le permite el dominio racional del mismo a través del trabajo o la formación.de la cosa. En el caso del señor, su autonegación implicaría la restricción de su voluntad o libertad natural, de manera tal que no sólo restituyese al siervo su condición de autoconciencia, sino que intuya que sólo en una autoconciencia libre puede acceder a la verdadera experiencia de su libertad. ${ }^{28}$

La realización cabal de esta relación entre sujetos libres, que supone la superación de la lucha como forma de su ser-reconocido, y, por ende,

${ }^{26}$ Hegel, Phänomenologie des Geistes, p. 133; v. cast. p. 118.

27 Cf. también para ello Enciclopedia de las Ciencias Filosóficas, $\iint 435-436$, en donde Hegel expone, precisamente en la figura del siervo, el valor ético que tiene la negación de sí para efectos de la conformación de la libertad absoluta del ser humano: la experiencia de la conciencia servil representa el "comienzo" de la vida ética.

28 "Sólo mediante el devenir libre del esclavo, el señor deviene también completamente libre": Enciclopedia de las Ciencias Filosóficas, \$ 436, "agregado". En la estructura de la Fenomenología del Espiritu, concretamente en el apartado de la Razón titulado "La realización de la autoconciencia racional por sí misma", Hegel expone el reconocimiento mutuo de las autoconciencias libres en el concepto de "pueblo", que entiende allí como "reino de la eticidad". Cf. Hegel, Phänomenologie des Geistes, pp. 233 y ss; v. cast. pp. 208 y ss. 
significa en Hegel la aparición del Estado, 29 es condición para la constitución de un tipo de comunidad de sujetos autoconscientes caracterizada por el mutuo intuirse de los mismos en sus respectivas singularidades o sí mismos independientes. Por eso Hegel dirá, ya al final de la exposición de la figura del deseo, justamente antes de la exposición de la dialéctica del reconocimiento, que en este ser de una autoconciencia para otra "está presente ya para nosotros el concepto del espíritu"30. Pues el "concepto del espíritu" no es otro que "esta sustancia absoluta, que, en la perfecta libertad e independencia de su contraposición, es decir, de distintas conciencias de sí que son para sí, es la unidad de las mismas". ${ }^{31}$

La dialéctica del deseo tiene así, en su momento culminante, la aparición del "concepto del espíritu", su justificación esencial en el marco experiencial de la figura de autoconciencia. Que el deseo de reconocimiento sea sólo la aparición de este concepto, del mundo ético propiamente dicho, evidencia el carácter conflictivo que caracterizará su propio proceso, en la medida en que éste, en su inmediatez, se dará en términos de lucha a muerte por el reconocimiento. Pero evidencia, asimismo, que es con la superación de tal proceso, superación que Hegel entiende en términos de "autonegación" de la singularidad hundida en el deseo, como, acaso paradójicamente, se aseguraría la satisfacción de tal deseo: éste, en efecto, no sería ya el pathos de un sí mismo necesitado de autoafirmación, sino expresión misma de la racionalidad característica del mundo ético, la racionalidad que encarnan sus instituciones. Éstas garantizarán no sólo la experiencia universal del reconocimiento en el mundo ético, sino que respetarán las experiencias particulares en que, dentro del mismo, se resuelve el fenómeno del reconocimiento, las esferas correspondientes a la familia, la sociedad civil y el Estado.

Recibido: 06/2012. Aceptado: 09/2012.

${ }^{29}$ Cf. Enciclopedia de las Ciencias Filosóficas, \433.

${ }^{30} \mathrm{Hegel}$, Phänomenologie des Geistes, p. 127; v. cast. p. 113.

${ }^{31}$ Idem. 